\title{
Germanica
}

\section{Le caractère de la propagande totale}

\section{Siegfried Kracauer}

Traducteur : Guillaume Plas

\section{(2) OpenEdition}

\section{Journals}

Édition électronique

URL : https://journals.openedition.org/germanica/8516

DOI : 10.4000/germanica.8516

ISSN : 2107-0784

\section{Éditeur}

Université de Lille

\section{Édition imprimée}

Date de publication : 1 juin 2020

Pagination : $37-50$

ISBN : 978-2-913857-45-2

ISSN : 0984-2632

Référence électronique

Siegfried Kracauer, "Le caractère de la propagande totale », Germanica [En ligne], 66 | 2ème trimestre 2020, mis en ligne le 02 janvier 2022, consulté le 08 janvier 2022. URL : http://

journals.openedition.org/germanica/8516 ; DOI : https://doi.org/10.4000/germanica.8516

(C) Tous droits réservés 


\section{Le caractère de la propagande totale}

Siegfried Kracauer

B. [Le caractère de la propagande totale]*

I.

La guerre est également à l'origine de la propagande dont il est ici question. Je précise par avance qu'en dépit de la conformité générale entre la pratique de la propagande en Allemagne et cette même pratique en Italie, c'est avant tout de la situation en Allemagne qu'il convient de déduire la théorie de la propagande totalitaire. Cela tient déjà à ce que le national-socialisme, dans son ascension vers le pouvoir, est confronté à des difficultés bien plus grandes que ne l'est le fascisme. Des difficultés qui ne peuvent être surmontées que de façon propagandistique ; pour ne rien dire du fait que la tendance allemande à ce que toute action soit motivée par une vision du monde oblige à mobiliser un nombre plus conséquent de moyens de propagande.

Quiconque veut accéder au pouvoir doit gagner les masses à sa cause. Suffit-il pour cela de recourir à la pure violence ? Hitler tire - de la guerre, paradoxalement - l'enseignement que pas même le pouvoir armé n'est en mesure de s'imposer ou de se maintenir par la seule violence, mais qu'il a au moins tout autant besoin d'exercer une influence spirituelle sur les

* - N. d. T. : Les ajouts entre crochets, dans le corps du texte ou en note, sont ceux de Bernd Stiegler, l'éditeur du texte allemand (qui a réalisé cette édition avec la collaboration de Joachim Heck et Maren Neumann) 
masses qu'il lui faut diriger. Dans Mein Kampf, après avoir recopié à la va-vite des extraits de ceux qui, parmi la presse allemande, tentaient de tempérer quelque peu l'assurance euphorique en la victoire, il poursuit ainsi à leur sujet :

Ils ne se doutaient pas un instant que l'enthousiasme, une fois bridé, ne pourrait plus être ravivé à loisir. Il est une ivresse et doit être maintenu dans cet état. Or, comment s'imaginaient-ils que puisse être surmontée sans ce pouvoir de l'enthousiasme l'épreuve d'un combat qui, mesuré à l'échelle de l'être humain, plaçait les qualités spirituelles de la nation devant les défis les plus immenses ${ }^{1}$ ?

Recourir au seul instrument de la violence apparaît à Hitler d'autant plus inapproprié qu'il lui faut non seulement conquérir les masses indifférentes, mais aussi celles empreintes de socialisme - des masses donc déjà sous l'influence d'une doctrine. Suite à des réflexions politiques qui témoignent de son instinct pour le pouvoir et la façon d'y accéder, il parvient à la conclusion suivante :

Des conceptions, des idées, de même que des mouvements reposant sur une base intellectuelle spécifique, peu importe que celle-ci soit fausse ou vraie, ne peuvent plus être brisés, à partir d'un certain stade de leur développement, à l'aide d'instruments de pouvoir de type technique que si ces armes corporelles sont en même temps elles-mêmes porteuses d'un nouveau concept mobilisateur, d'une idée ou vision du monde 2 .

«[...] peu importe que celle-ci soit fausse ou vraie [...]» : rien de plus révélateur que cette incise, à laquelle Mussolini aurait souscrit. Elle exemplifie tout le nihilisme de son auteur; elle démontre en toute concision que le but d'Hitler n'est nullement de détourner les masses d'une opinion incorrecte pour les mener à une opinion correcte, mais que seul lui importe, plus fondamentalement, de tenir les masses sous son emprise spirituelle. Cela est parfaitement logique, dans la mesure où, lorsque c'est le pouvoir en soi que l'on désire, la teneur de vérité de la doctrine que l'on adopte et défend dans l'intérêt de ce désir n'a pas la

1. - [« Man hatte keine blasse Ahnung, daß die Begeisterung, erst einmal geknickt, nicht mehr nach Bedarf zu erwecken ist. Sie ist ein Rausch und ist in diesem Zustande weiter zu erhalten. Wie aber sollte man ohne diese Macht der Begeisterung einen Kampf bestehen, der nach menschlichem Ermessen die ungeheuersten Anforderungen an die seelischen Eigenschaften der Nation stellen würde ? », Adolf Hitler, Mein Kampf, t. 1 : Eine Abrechnung, München, F. Eher, 1934, p. 183.]

2. - [" Vorstellungen und Ideen sowie Bewegungen mit bestimmter geistiger Grundlage, mag diese nun falsch sein oder wahr, können von einem gewissen Zeitpunkt ihres Werdens an mit Machtmitteln technischer Art nur mehr dann gebrochen werden, wenn diese körperlichen Waffen, zugleich selber Träger eines neuen zündenden Gedankens, einer Idee oder Weltanschauung sind », ibid., p. 186 sq.] 
moindre importance. Certes, pour parvenir à avoir les masses sous son contrôle, on doit donner l'impression d'avoir une idée que l'on cherche à propager. Mais ce qui importe, ce n'est pas que la propagande se mette au service des idées que l'on aura identifiées comme vraies ; c'est que la propagande s'entende à se servir d'idées, quelles qu'elles soient. L'idée mobilisatrice est l'un des instruments de la propagande, et non inversement la propagande l'instrument de l'idée. Dès le départ, Hitler se focalise sur la technique de propagande en faisant abstraction du contenu que celle-ci sert à propager ; c'est-à-dire sur la technique de l'influence des masses en tant que telle.

Hitler revient de la guerre non seulement avec l'enseignement qu'une telle influence est nécessaire, mais aussi avec un modèle : celui de la propagande de guerre déployée par l'Entente : « J'ai moi aussi appris infiniment à étudier cette propagande de guerre ennemie $»^{3}$, confesse-t-il au début d'un chapitre consacré à son analyse - une véritable analyse d'expert, dont il met en usage les conclusions dans sa propre pratique. La paix est pour lui la continuation de la guerre par d'autres moyens. Il attribue la défaite allemande à l'écrasante supériorité de la propagande anglaise et américaine ; à cet égard, la capacité de celle-ci à faire en sorte que l'invraisemblable apparaisse comme la vérité suscite tout particulièrement son admiration. « Au début, elle donnait l'impression d'être insensée, par l'insolence de ses affirmations ; plus tard, elle devint désagréable ; et pour finir, on la crut $»^{4}$. Hitler ne se préoccupe pas du contenu de la propagande, mais conçoit celle-ci exclusivement comme un moyen dont l'usage est promesse de pouvoir : en atteste l'angle exclusivement esthétique sous lequel il formule son appréciation de la propagande social-démocrate. Sans prendre en considération le fait qu'elle émane d'une doctrine précise - « peu importe que celle-ci soit fausse ou vraie »-, il la détache de la cause qu'elle représente et étudie, à l'aide de ce modèle ainsi isolé, les méthodes les plus efficaces pour capter les individus.

Ce qui a gagné les millions de travailleurs au marxisme, c'est moins la manière d'écrire des pères de l'église marxiste que le travail de propagande infatigable et proprement monumental réalisé par les dizaines de milliers d'agitateurs politiques infatigables, depuis le grand prédicateur jusqu'à l'employé de syndicat subalterne et au délégué prenant la parole lors de débats ; ce sont les centaines de milliers de réunions lors desquelles, dans des auberges enfumées, ces tribuns, debout sur des tables, haranguèrent les masses et purent ainsi acquérir une connaissance fabuleuse de ce matériau humain, ce qui les mit plus à même encore de choisir les armes

3. - [ «An dieser feindlichen Kriegspropaganda habe auch ich unendlich gelernt », ibid., p. 193.]

4. - [ [ Sie war im Anfang scheinbar verrückt in der Frechheit ihrer Behauptungen, wurde später unangenehm und ward endlich geglaubt. », ibid., p. 203.] 
les plus propices à attaquer les fortifications de l'opinion publique. Et ce sont, également, les gigantesques manifestations de masse $[\ldots]^{5}$.

En somme, la naissance de la propagande totalitaire est le fruit de la volonté de pouvoir de la clique de l'après-guerre. Toutes les définitions de cette propagande la conçoient comme un procédé permettant à ceux qui l'utilisent de prendre possession des êtres humains. « La propagande [...] », explique Goebbels, « n'a qu'une finalité ; une finalité qui a toujours pour nom en politique : conquête de la masse $»^{6}$. D'où l'intérêt passionné qu'éprouve Hitler pour la propagande. Dans la nuit du putsch manqué de Munich, un conseil de guerre eut lieu auquel participèrent, entre autres, Ludendorff et Göring, et tout au long de la concertation Hitler ne cessa de s'exclamer : «La propagande ! La propagande ! Désormais, ce qui importe, ce n'est rien d'autre que la propagande $\gg^{7}$ !

\section{II.}

L'une des tâches de toute propagande, et non des moindres, est d'écarter les obstacles qui s'opposent à la croyance en l'objectif que l'on cherche à propager. Et du type d'opposition rencontrée dépend la forme à donner à la propagande elle-même. Les nationaux-socialistes, que l'on prendra ici comme exemple, désirent un pouvoir qui consisterait dans le contrôle d'un appareil de pouvoir organisé comme en situation de guerre. D'un tel appareil de pouvoir, le présupposé fondamental est l'unité nationale. Or, quelle image leur offre la situation ? Celle d'une opposition entre classes, d'une fracturation en de multiples partis. Bien sûr, les nationaux-socialistes, tout comme les fascistes, voient dans le bolchévisme et, de manière plus générale, dans les partis « marxistes » leur adversaire principal, tandis qu'ils entretiennent d'assez bonnes relations avec ce que l'on a coutume d'appeler les partis nationaux.

5. - [ « Was dem Marxismus die Millionen von Arbeitern gewonnen hat, das ist weniger die Schreibart marxistischer Kirchenväter als vielmehr die unermüdliche und wahrhaft gewaltige Propagandaarbeit von Zehntausenden unermüdlicher Agitatoren, angefangen vom großen Hetzapostel bis herunter zum kleinen Gewerkschaftsbeamten und zum Vertrauensmann und Diskussionsredner; das sind die Hunderttausende von Versammlungen, bei denen in qualmender Wirtsstube auf dem Tische stehend, diese Volksredner auf die Massen einhämmerten und so eine fabelhafte Kenntnis dieses Menschenmaterials zu gewinnen wußten, was sie erst recht in die Lage versetzte, die richtigsten Angriffswaffen auf die Burg der öffentlichen Meinung zu wählen. Und das waren weiter die gigantischen Massendemonstrationen [...]», ibid., p. 59 sq.]

6. - [« Die Propaganda [...] hat nur ein Ziel ; und zwar heißt dieses Ziel in der Politik immer : Eroberung der Masse », Joseph Goebbels, Kampf um Berlin, t. 1 : Der Anfang, München, F. Eher, 1935, p. 18.]

7. - [«Propaganda, Propaganda, Propaganda, es kommt jetzt nur auf Propaganda an ! », citation d'après le souvenir de Heiden, in : Konrad Heiden, Adolf Hitler, t. 1 : Das Zeitalter der Verantwortungslosigkeit, Zürich, Europa Verlag, 1936, p. 109.] 
Cependant, cette différenciation opérée selon le critère du contenu varie en fonction des circonstances, qui peuvent par exemple conduire à ce que le Zentrum soit considéré comme un ennemi pire encore que le SPD ; et elle ne doit pas masquer le fait que c'est dans l'existence même de partis politiques que cette clique qui aspire au pouvoir voit le mal véritable. À cet égard, le Deutschnationale Volkspartei n'a pas de meilleures cartes que le Parti communiste.

Le système des partis ne rend pas seulement plus ardu le rassemblement resserré des forces de la nation, il empêche le travail de propagation de la tendance qui y aspire.

Pourquoi les cliques national-socialistes et fascistes prennent-elles dès le départ pour cible le système des partis ? Non seulement parce que celui-ci rend plus ardu le rassemblement resserré des forces de la nation, mais aussi, et plus encore, parce qu'il fait obstacle au travail de propagation de l'ambition de pouvoir qui anime ces cliques. La bohème national-socialiste doit se rendre à l'évidence que des préempteurs d'une partie de l'électorat sont déjà à l'œuvre dans l'essentiel des partis. Le Zentrum a les catholiques sous son contrôle ; les organisations prolétariennes ont la mainmise sur les travailleurs et sont, du fait de leur poids politique, sans cesse en mesure d'exercer une force d'attraction sur les chômeurs et les couches prolétarisées ; les partis bourgeois ont eux aussi un nombre non négligeable de partisans. Reste-t-il de quelconques masses disponibles? " De tous les côtés, on entend le cri de guerre, les catholiques, les protestants, les Bavarois, les Prussiens, les bourgeois, les prolétaires », s'exclame encore Goebbels le 31 juillet 1932 lors d'un discours de campagne à Munich :

À croire qu'il n'y a plus d'Allemands en Allemagne. [...] Elles [les puissances internationales] se sont mises à leur aise sur nos dos sanglants, la nation consume ses forces en son sein et n'est plus ni disposée, ni en mesure, d'en faire usage à l'extérieur. Voilà la conséquence de cette maudite politique de parti ${ }^{8}$.

Qui plus est, les partis sont en possession des idées qui excitent les masses. Or, la clique national-socialiste est doublement tributaire de telles idées mobilisatrices ; car sa volonté nihiliste de pouvoir, qui constitue sa motivation décisive, ne fait pas un bon slogan de campagne. Cette clique

8. - [« Überall ertönt das Kampfgeschrei, die Katholiken, Protestanten, die Bayern, die Preußen, die Bürger, die Proletarier. Man muß ja zur Meinung kommen, es leben in Deutschland keine Deutschen mehr. - Sie haben sich auf unseren blutenden Rücken eingerichtet, die Nation verbraucht ihre Kraft im Innern und ist nicht mehr gewillt und nicht mehr fähig, Kraft nach außen einzusetzen. Das ist die Folge der verruchten Parteipolitik », J. Goebbels, Discours de campagne du 31 juillet 1932 à Munich, in : Id., Revolution der Deutschen, 14 Jahre Nationalsozialismus, Oldenburg, Stalling, 1933, p. 101 sq.] 
s'est certes autodéclarée la protectrice naturelle de l'idée nationale, qui, au moins, emporte l'enthousiasme de certains pans de la masse ; mais cette idée n'est pas sa propriété, elle fait partie du patrimoine de la droite. Quant à l'idée de socialisme, elle aurait bien du mal à nier au cours des années où la propagande national-socialiste se développe qu'elle est défendue, si ce n'est par le SPD, à tout le moins par le Parti communiste. À ces attaches organisationnelles des idées dont la propagande nationalsocialiste pourrait se servir s'ajoutent des attaches dans la sphère idéelle, qui se recoupent souvent avec celles organisationnelles. Le nationalisme prend des airs qui excluent qu'il puisse faire usage de la puissance de traction du socialisme ; le socialisme entretient une liaison durable avec le pacifisme, ou du moins mine-t-il par le simple fait de son internationalité le concept de nation. Et d'autres relations du même genre se nouent dans les annexes / territoires coloniaux des grandes idées publicitaires. Ainsi la famille est-elle à la fois le refuge de la nation et de la religion. Et la religion a quant à elle certes coutume de soutenir l'appel du national, mais elle fait signe vers un au-delà de la nation.

La difficulté la plus grande que rencontre la propagande nationalsocialiste ou fasciste ne réside cependant pas même dans les attaches que l'on vient de nommer à des partis ou des idées; elle réside bien plutôt dans le fait que les idées qui excitent les masses trouvent leurs racines dans des intérêts. En effet, ces idées non seulement ne sont pas libres ; elles sont aussi les dérivés, ou les coquilles idéologiques, d'intérêts vitaux qui s'organisent et luttent entre eux, précisément, sous la forme de partis. Les intérêts capitalistiques s'adjoignent l'idée nationale, et le socialisme est l'intérêt de ceux qui sont ou devraient être à la hauteur des finesses du texte originel marx[ien]. Étant entendu qu'à long terme, les intérêts sont le levier le plus puissant, la force mobilisatrice des idées dont il est ici question s'explique par conséquent moins par leur contenu purement idéel que par leur ancrage dans des intérêts. Or, s'il en est ainsi, il n'apparaît alors possible d'exercer une influence sur des masses qu'à la condition que l'on représente les intérêts des masses en question; et on ne peut alors éviter, du fait des conflits d'intérêts entre les masses, d'avoir toujours une partie d'entre elles contre soi si l'on aspire à gagner l'autre. Il n'est alors pas envisageable d'abolir la lutte des classes et d'unifier les masses en un seul et même ensemble autrement qu'en procédant à une intervention chirugicale sur la sphère des intérêts.

\section{III.}

Les partis sont soumis à ces contraintes manifestes : ce sont des édifices rigides, qui se sont voués à des intérêts spécifiques, et dont la propagande est mise au service de ces intérêts respectifs. Ce qu'Hitler, dont l'instinct se porte sur l'édification et le contrôle de l'appareil de 
pouvoir national, veut quant à lui dès le départ conquérir, ce sont les masses en tant que telles. Cela signifie qu'il doit chercher à mobiliser les intérêts des masses, intérêts qui, du fait que leur agencement suit celui des partis, s'opposent les uns aux autres. Il n'aurait rien à gagner à se faire le parti de quelconques intérêts particuliers et, ainsi, à faire du national-socialisme un parti parmi d'autres. Son exclamation « La propagande! La propagande ! Désormais, ce qui importe, ce n'est rien d'autre que la propagande ! » traduit justement que ce qui lui importe, ce n'est pas de défendre des intérêts, mais d'influencer les masses, quels que soient leurs intérêts. Dès lors, au lieu de pénétrer dans la sphère des intérêts et de s'efforcer par exemple d'annuler effectivement les oppositions entre les classes, il laisse persister les causes des oppositions entre les classes et appréhende plutôt ces oppositions comme un phénomène, qu'il s'agit de surmonter en tant que phénomène. C'est ce qui explique que le socialisme et le capitalisme, que le travailleur et l'entrepreneur soient des facteurs qu'il prend d'égale manière en compte. Il se meut dans la sphère de l'apparence et du reflet des forces sociales réelles, et l'image-symptôme est à ses yeux la chose dernière - une chose qui, en tout cas, le préoccupe davantage que l'origine de cette image. C'est ce qu'Ortega y Gasset fait remarquer lorsque, [observant] la pensée bourgeoise [depuis] son point de vue de démocrate convaincu, il décrit «l'homme des masses » fasciste de la manière suivante :

Ce qu'il fait, il le fait «comme si », de la même façon que le fils de famille fait ses bêtises. Tout le zèle qu'il met à adopter dans chaque situation de son existence une attitude tragique, décisive, dans laquelle tout son destin est en jeu, est une apparence vide. Il joue à la tragédie, parce qu'il tient la véritable tragédie pour peu vraisemblable dans notre monde civilisé9 .

Cette volonté d'Hitler et des siens de minimiser le rôle des intérêts transparaît dans toutes leurs déclarations : «Intégrer [...] notre peuple dans toute sa diversité au sein d'une communauté du peuple nationale, explique Hitler, ne signifie pas renoncer à porter des intérêts d'états (Stand) lorsque ceux-ci sont justifiés. Des divergences entre les intérêts respectifs d'états et de corps de métier, ce n'est pas la même chose qu'une scission entre classes $»^{10}$. Lui importe avant tout le développement des intérêts au profit des idées. Ainsi affirme-t-il, à rebours de la réalité :

9. - [ « Was er macht, macht er "als ob”, wie der Familiensohn seine Dummheiten. Aller Eifer, mit dem man bestrebt ist, in jeder Lebenslage tragische, letzte, schicksalsschwere Haltungen einzunehmen, ist leerer Schein. Man spielt Tragödie, weil man die wirkliche Tragödie in unserer zivilisierten Welt für unwahrscheinlich hält », José Ortega y Gasset, Der Aufstand der Massen, Stuttgart, Deutsche Verlags-Anstalt, 1930, p. 77.]

10. - [« Die Eingliederung der [...] breiten Masse unseres Volkes in eine 
En 1914, tant que le peuple allemand croyait encore se battre pour des idéaux, il tint bon ; à partir du moment où on le fit combattre pour son pain quotidien, il préféra abandonner. Nos « hommes d'État» pleins d'esprit s'étonnèrent de ce changement de mentalité. Ils ne comprirent jamais qu'à partir du moment où un homme se bat pour un intérêt économique, il évite autant que faire se peut de mourir, car lui échapperait alors à jamais la jouissance du salaire de son combat ${ }^{11}$.

Goebbels lui aussi n'a que mépris pour les intérêts, lorsqu'il ne préfère les flatter : « ils ont mobilisé les porteurs d'intérêts les uns contre les autres, déclare-t-il au sujet des partis dans le discours électoral de Munich déjà cité, ils ont réveillé les bas instincts $\rangle^{12}$. Et Göring s'exprime de manière similaire le 9 avril 1933 : « De la même façon que le socialisme [a été] abaissé par le marxisme, qui en a fait une terne question de salaire et d'estomac, le monde des partis bourgeois a abaissé le nationalisme pour en faire ce terne patriotisme d'acclamation qui trouve ses racines dans l'alcool et le portemonnaie ${ }^{13}{ }^{13}$. À cet égard, la critique formulée par Georg Strasser, à l'époque des obligations en dollars et des procèsspectacles, à l'encontre des partis politiques - critique visant à révéler la dépendance des idées à l'égard des intérêts - est particulièrement instructive : «L'industrie, et tout particulièrement l'industrie lourde, dit-il à l'occasion d'une analyse de la Deutsche Volkspartei,

est liée à l'espace, au sol, qui est national ; elle entretient un rapport de dépendance réciproque avec ses travailleurs, d'où découle que sa représentation politique, le Deutsche Volkspartei, éprouve de l'intérêt et de la compréhension pour les exigences d'une politique nationale, et de l'intérêt et de la compréhension pour une politique sociale étendue [...]. Cela ne doit pas empêcher de percevoir que l'économie [...] ne se

nationale Volksgemeinschaft bedeutet keinen Verzicht auf die Vertretung berechtigter Standesinteressen. Auseinandergehende Standes- und Berufsinteressen sind nicht gleichbedeutend mit Klassenspaltung [...] », A. Hitler, Mein Kampf, t. 1, op. cit., p. 372.]

11. - [ « Solange das deutsche Volk im Jahre 1914 noch für Ideale zu fechten glaubte, hielt es stand ; sowie man es nur mehr um das tägliche Brot kämpfen ließ, gab es das Spiel lieber auf. Unsere geistvollen "Staatsmänner" aber staunten über diesen Wechsel der Gesinnung. Es wurde ihnen niemals klar, daß ein Mensch von dem Augenblick an, in dem er für ein wirtschaftliches Interesse ficht, den Tod möglichst meidet, da ja dieser ihn um den Genuß des Lohnes seines Kampfes für immer bringen würde », ibid., p. 168.]

12. - [ « sie haben die Interessenten gegeneinander mobilisiert, sie haben die niederen Instinkte wachgerufen », J. Goebbels, Discours de campagne du 31 juillet 1932 à Munich, in : Id., Revolution der Deutschen, op. cit., p. 101.]

13. - [« So wie der Sozialismus vom Marxismus [herab]degradiert [war] zu einer öden Lohn- und Magenfrage, so hat die bürgerliche Parteienwelt den Nationalismus degradiert zu dem öden Hurra-Patriotismus, dessen Wurzeln der Alkohol und das Portemonnaie gewesen sind », Hermann Göring, « Nationalismus und Sozialismus », discours du 9 avril 1933 à l'occasion de l'appel général des SA au Palais des sports de Berlin, in : Id., Reden und Aufsätze, éd. par Erich Gritzbach, München, F. Eher, 1938, p. 37-50, ici p. 38.] 
fait la représentante des exigences nationales que tant que ses propres [...] intérêts économiques vont dans la même direction! Et qu'elle s'oppose aux revendications sociales dès l'instant où celles-ci entravent la recherche du profit ${ }^{14}$.

Une telle analyse correspond peu ou prou à l'analyse marxiste, à la différence près que là où le marxiste l'effectue dans le but de démystifier des contenus pseudo-idéels, Strasser y procède afin de montrer que le pouvoir magique des idées souffre malheureusement du handicap que constituent les intérêts de parti. «Leur dévotion à l'idée nationale est [...] authentique $»^{15}$, remarque-t-il au sujet des membres du Deutschnationale Volkspartei, mais celui-ci « aussi s'est voué au système économique capitaliste $»^{16}$. Et comme il ne peut nier que l'intérêt des partis des travailleurs est que soit approuvée l'idée du socialisme, il leur reproche de dégrader cette idée en en faisant un intérêt de classes internationales ; sans parler du fait qu'il accuse le SPD de s'être embourgeoisé et d'avoir trahi l'idée.

Mais tous les efforts entrepris pour désavouer les intérêts ne peuvent cacher à la face du monde que celui-ci est, dans des circonstances normales, régi par des intérêts. Dès lors, Hitler n'a plus d'autre solution que d'attirer les masses à lui avec le seul appui de la propagande, puisque l'intérêt qui le meut ne se recoupe avec aucun intérêt social majeur. Il en découle pour la propagande national-socialiste (et fasciste) une tâche qui paraît irréalisable, pour ne pas dire chimérique : celle de libérer de toute attache les idées fascinantes susceptibles d'être utilisées pour de la propagande, afin de pouvoir les manipuler à sa guise. Autrement dit, ces idées doivent être déprises de leur lien à des idées adverses et, surtout, arrachées aux intérêts dans lesquels elles sont solidement enracinées. On pourrait objecter à cela que le national-socialisme parvient effectivement à contenter les masses en promettant aux différents groupes de la population la satisfaction simultanée ou successive de leurs intérêts divergents,

14. - [ « Die Industrie und gerade die Schwerindustrie ist gebunden an den Raum, an den Boden, der national ist ; ist in einem wechselseitigen Abhängigkeitsverhältnis zu ihrer Arbeiterschaft, woraus sich ergibt, daß die politische Vertretung, die Deutsche Volkspartei, Interesse und Verständnis für die Erfordernisse einer nationalen Politik hat und Interesse und Verständnis für eine weitgehende Sozialpolitik [...]. Das darf nicht hindern zu erkennen, daß die Wirtschaft [...] die nationalen Erfordernisse nur bis zu dem Punkt vertritt, bis zu dem ihre eigenen [...] wirtschaftlichen Interessen parallel damit gehen! Und daß sie die sozialen Forderungen sofort bekämpft, wenn sie an das Gewinnstreben stoßen », Georg Strasser, « Die deutsche Volkspartei », in : Id., Kampf um Deutschland. Reden und Aufsätze eines Nationalsozialisten, München, F. Eher, 1932, p. 206-213, ici p. 209.]

15. - [ [ Ihre Hingabe an die nationale Idee ist [...] echt», G. Strasser, « Wir und die Anderen ", in : Id., Kampf um Deutschland, op. cit., p. 62-72, ici p. 67.]

16. - [ « aber auch die Deutschnationale Volkspartei ist dem kapitalistischen Wirtschaftssystem verfallen », ibid., p. 68.] 
[une audacieuse tentative de tromperie à laquelle il procède bel et bien,] mais qui n'a de chances de fonctionner que si l'on s'est auparavant déjà occupé de déserrer par la propagande les liens entre les intérêts et les idées. Si tel ne devait être le cas, alors la tromperie serait à son tour inévitablement démasquée comme tromperie et demeurerait sans effet.

Ce que l'on définit ici comme la tâche de la propagande, Gregor Strasser l'énonce dans l'un de ses discours avec une clarté inégalable : " Ainsi se forma peu à peu ", affirme-t-il là au sujet des partis allemands de l'après-guerre,

l'un de ces fronts doublement faux : nationalisme plus capitalisme contre internationalisme plus socialisme. Tous deux ont d'égale manière pour enjeu le combat du peuple allemand pour sa liberté. Et comme dans chacun des deux camps, une moitié a raison [...], on rencontre dans chacun une égale proportion de dévouement, de disposition au sacrifice, de volonté de se battre et de confiance en la victoire ! Il s'agit donc, «à droite », de délivrer le nationalisme de sa liaison funeste avec le capitalisme, et, " à gauche », de délivrer le socialisme de sa liaison funeste avec l'internationalisme ${ }^{17}$.

Les mots d'ordre des partis, qui sont leurs idéologies, sont chevillés aux intérêts dont ces partis sont les représentants, si bien que chaque changement d'orientation provoqué par les intérêts entraîne avec lui de façon décisive une modification du positionnement idéel ; dès lors, on peut aussi comprendre de façon générale la propagande national-socialiste et fasciste comme une tentative de dynamiser le système figé au sein duquel celle-ci prend forme. Ce n'est qu'en faisant dérailler ce système, en démolissant son stable édifice et en en mettant les débris sens dessus dessous qu'elle s'ouvre, de son propre fait, la possibilité de faire ses preuves et de gagner en influence. «Hitler, le secoueur, des consciences l'agitateur / De l'âme de notre peuple le vrai transmetteur $»^{18}$, rime l'un des partisans d'Hitler à l'époque où celui-ci séjourne à la prison de Landsberg. Et Ernst Krieck fait au sujet du national-socialisme la remarque suivante : «En tant que mouvement de masse, il présuppose

17. — [ So entstand allmählich eine [...] jener doppelt falschen Fronten : Nationalismus plus Kapitalismus gegen Internationalismus plus Sozialismus. Beiden gilt gleichmäßig der Freiheitskampf des deutschen Volkes. Gerade weil in beiden die Hälfte richtig [...] ist, gerade deshalb sind in beiden Heerlagern ja auch gleichmäßig vertreten Hingabe, Opferwilligkeit, Kampfwille und Siegeszuversicht ! Es gilt daher von "rechts" den Nationalismus zu lösen aus der unheilvollen Verbindung mit dem Kapitalismus ; von "links" den Sozialismus zu lösen aus der verhängnisvollen Verbindung mit dem Internationalismus », G. Strasser, « Nationalsozialismus und Geschichte », discours du 1 er janvier 1929 au Reichstag à Berlin, in : Id., Kampf um Deutschland, op. cit., p. 225-229, ici p. 225.]

18. - [« Hitler, Schüttler, Seelenrüttler / Unseres Volkstums wahrer Mittler », K. Heiden, Adolf Hitler, t. 1, op. cit., p. 209.] 
l'art d'exciter la masse : la masse doit devenir fluide si l'on veut pouvoir lui donner forme $»^{19}$. Fluidifier ce complexe solide sous la forme duquel la superstructure idéelle et ses fondements ont été soudés l'une avec les autres, ou, pour le dire autrement, opérer la transition de rapports sociaux statiques vers des rapports sociaux dynamiques : voilà précisément la tâche à laquelle est confrontée la propagande national-socialiste et fasciste. Il lui faut mettre en mouvement l'inamovible ; ce pourquoi également le national-socialisme et le fascisme se donnent, à juste titre, le nom de « mouvement ». "C'est la raison pour laquelle le nationalsocialisme n'est ni parti, ni programme - explique Krieck dans son style aussi lisse qu'enflé -, mais mouvement fluide et fluidifiant, qui certes, un jour, lorsqu'il aura vaincu, débouchera sur une nouvelle forme, un nouvel ordre, une nouvelle ratio, mais qui doit demeurer, pur mouvement qu'il est, à l'état de fluide jusqu'à ce qu'il ait saisi et imprégné dans son entier l'espace vital de notre peuple $»^{20}$. Lorsque, par la suite, Mussolini entreprit de se lier avec les propriétaires terriens, il ne perdit pas une occasion de déclarer « que le fascisme ne constitue pas un nouveau parti mais le mouvement d'un front antibolchévique unitaire $»^{21}$. Hitler, quant à lui, énonce tout bonnement : « Mener signifie : savoir comment mouvoir les masses $»^{22}$, et il veille à ce que le parti, même après sa prise de pouvoir, demeure l'élément mouvant. Aussi Robert Pelloux, dans son excellente étude Le Parti national-socialiste et ses rapports avec l'État, peut-il expliquer dans un passage excentré « que le parti ne se substitue pas à l'Etat, mais qu'il lui apporte l'impulsion, le dynamisme nécessaire $»^{23}$.

Ce mouvement, dont la fonction est de désencombrer les idées afin de leur donner une orientation nouvelle, n'est par conséquent pas, ou si peu, mouvement des intérêts réels en place. Cela n'empêche certes pas qu'il se développe, par la force des circonstances, dans une direction favorable au capitalisme alors en danger. Le fait qu'il le « sauve » ne suffit néanmoins pas à le caractériser comme un mouvement qui aurait

19. — [«Als Massenbewegung setzt er voraus, die Kunst der Massenerregung : Masse muß flüssig werden, wenn sie gestaltet sein soll », Ernst Krieck, Nationalpolitische Erziehung, Leipzig, Armanen-Verlag, 1937, p. 36.]

20. - [« Darum ist Nationalsozialismus auch nicht Partei und Programm, sondern flüssige und flüssigmachende Bewegung, die wohl einst mit dem Sieg in neue Form, Ordnung, Ratio einmünden wird, die sich aber als reine Bewegung solange in Fluß halten muss, bis sie das Ganze unseres völkischen Lebensraumes ergriffen und durchdrungen hat », ibid., p. 38.]

21. - [ « daß der Faschismus keine neue Partei, sondern die Bewegung einer antibolschewistischen Einheitsfront darstelle », Ignazio Silone, Der Fascismus. Seine Entstehung und seine Entwicklung, Zürich, Europa-Verlag, 1934, p. 85.]

22. - [« Führen heißt : Massen bewegen können », Adolf Hitler, Mein Kampf, t. 2 : Die nationalsozialistische Bewegung, München, F. Eher, 1934, p. 650.]

23. - [Robert Pelloux, Le Parti national-socialiste et ses rapports avec l'État, éd. par le Centre d'études de politique étrangère, Paris, Paul Hartmann, 1936, p. 82.] 
été formé spécifiquement dans le but de porter des intérêts capitalistes ; cela indique seulement qu'il se sert de ceux-ci pour s'emparer du pouvoir et exercer sa domination. Peut-être le capitalisme ne pouvait-il échapper à la menace et être préservé, en Allemagne comme en Italie, qu'à l'aide d'un ordre créé par autre que lui. Peut-être, aussi, le capitalisme ne fait-il en partie que s'imaginer être en danger, à force de se l'entendre dire. Toujours est-il que la structure du mouvement n'est pas celle d'un parti d'intérêts. Il se sert des capitalistes et les laisse se servir de lui, mais sans être leur porte-voix. Le fait qu'il doive à un moment donné ne faire qu'un avec eux est une autre chose, indépendante de sa spéculation au sujet de leurs intérêts. Mais si cela est vrai, alors ne s'effectue-t-il pas dans la sphère des intérêts. Et s'il ne s'effectue pas dans la sphère des intérêts, alors est-il nécessaire de préciser encore l'intention de la propagande. Celle-ci ne peut mener au but souhaité qu'à la condition qu'elle trouve un moyen et une manière de faire apparaitre le processus de dynamisation qu'elle a engendré comme un processus effectif. Qu'elle n'ait d'autre choix que de s'imposer une telle exigence, c'est ce que met en lumière la confrontation de deux déclarations en provenance de directions opposées. Tandis que l'antifasciste Silone, qui prend les intérêts sociaux comme grille d'analyse, définit la conséquence des idéologies fascistes comme la tentative de « noyer le langage irrépressible des faits dans un flot de paroles $»^{24}$, Goebbels, passant tous les intérêts sous silence, perçoit la mission de la propagande dans le fait de « gagner les individus à une idée d'une façon assez profonde, assez vivace pour qu'ils finissent par s'y abandonner entièrement et ne puissent s'en détacher ${ }^{25}$. La propagande national-socialiste et fasciste doit donc viser à ce que les individus s'abandonnent en fin de compte non aux faits, mais à « un flot de paroles »; à travailler la superstructure idéelle, après l'avoir arrachée aux intérêts qui constituaient son fondement, de telle sorte que ce soit elle qui serve de fondement sous-tendant des intérêts ; à ce que l'illusion apparaisse comme la réalité, et l'apparence, comme l'être lui-même.

Nous ne serions pas des socialistes si nous ne combattions pas la domination de classe du système capitaliste [...] et nous ne serions pas des nationalistes si nous ne repoussions pas avec la même passion chaque tentative haineuse de s'opposer à ce qu'une masse brutale de citoyens jusqu'à présent défavorisés inverse tout simplement ce jeu [...]! Voilà

24. - [ [ die nicht zu unterdrückende Sprache in einem Schwall von Worten zu ersticken », I. Silone, Der Fascismus, op. cit., p. 252.]

25. - [« Menschen für eine Idee zu gewinnen, so innerlich, so lebendig, daß sie am Ende ihr verfallen sind und nicht mehr davon loskommen », J. Goebbels, Discours aux directeurs des théâtres allemands du 8 mai 1933 à Hambourg, in : Erwin Leiser, " Deutschland erwache! » Propaganda im Film des Dritten Reichs, nouvelle édition augmentée, Reinbek, Rowohlt, 1978, p. 46.] 
précisément la chose la plus profonde dont nous avons pris conscience : que le socialisme véritable est identique au nationalisme véritable, et qu'il est tout autant l'ennemi mortel de la domination de classe [d'une bourgeoisie privilégiée que celui de la domination de classe du prolétariat.] $]^{26}$

La finalité de montages idéels comme celui-ci, que Georg Strasser - et il n'est pas le seul - échafaude à tour de bras, est qu'ils germent, par la force de dispositions particulières de la propagande, avec une puissance telle que la « masse brutale » finisse par ne plus savoir ce qui lui arrive.

« La propagande est un art »27, dit Goebbels. À l'aide de quels stratagèmes la propagande national-socialiste et fasciste entreprend-elle de réaliser ce qui paraissait impossible : disjoindre non seulement les idées des intérêts, mais aussi, dans le plein élan du mouvement qu'elle met en scène, imposer sa propre idée mobilisatrice, ou son propre assemblage idéologique mobilisateur, comme l'intérêt prépondérant, au regard duquel les intérêts réels passent au second plan ? La réponse est la suivante : puisqu'elle ne se fond elle-même dans aucun intérêt de classe, il ne lui reste pas d'autre solution que de modifier la structure psycho-physique de l'être humain. Mais avant d'analyser les méthodes par lesquelles elle s'efforce d'opérer cette modification, il est nécessaire de présenter les traits de caractère qui sont les siens, conformes à la manière dont elle est appliquée.

Traduit de l'allemand par Guillaume Plas

26. - [ « Wir wären keine Sozialisten, wenn wir nicht gegen die Klassenherrschaft des kapitalistischen Systems kämpfen würden [...] und wir wären keine Nationalisten, wenn wir nicht mit gleicher Leidenschaft jeden hassvollen Gegenversuch zurückweisen würden, daß eine brutale Masse bisher benachteiligter Staatsbürger dieses Spiel einfach umkehrt [...] ! Das gerade ist unser tiefstes Erkennen : daß der wahrhafte Sozialismus identisch ist mit dem wahrhaften Nationalismus und ebenso Todfeind ist der Klassenherrschaft [eines bevorrechtigten Bürgertums wie der Klassenherrschaft des Proletariats]», G. Strasser, « Bürger oder Proletarier », in : Id., Kampf um Deutschland, op. cit., p. 162-166, ici p. 165.]

27. - [ « Propaganda ist eine Kunst», J. Goebbels, Discours lors du Reichsparteitag, au Congrès des Gauleiter et des directeurs de la propagande le 16 septembre 1935 à Nuremberg, in : Helmut Heiber (éd.), Goebbels-Reden, t. 1 : 1932-1939, Düsseldorf, Droste, 1971, p. 219-228, ici p. 232.] 
